\title{
Critical role for CXCR2 and CXCR2 ligands during the pathogenesis of ventilator-induced lung injury
}

\author{
John A. Belperio, ${ }^{1}$ Michael P. Keane, ${ }^{1}$ Marie D. Burdick, ${ }^{1}$ Vedang Londhe, ${ }^{2}$ Ying Ying Xue,${ }^{1}$ \\ Kewang Li, ${ }^{1}$ Roderick J. Phillips, ${ }^{1}$ and Robert M. Strieter ${ }^{1,3}$ \\ ${ }^{1}$ Department of Medicine, Division of Pulmonary and Critical Care Medicine, \\ ${ }^{2}$ Department of Pediatrics, and \\ ${ }^{3}$ Department of Pathology and Laboratory Medicine, David Geffen School of Medicine at University of California, Los Angeles, \\ Los Angeles, California, USA
}

\begin{abstract}
Mortality related to adult respiratory distress syndrome (ARDS) ranges from $35 \%$ to $65 \%$. Lung-protective ventilator strategies can reduce mortality during ARDS. The protective strategies limit tidal volumes and peak pressures while maximizing positive end-expiratory pressure. The efficacy of this approach is due to a reduction of shear-stress of the lung and release of inflammatory mediators. Ventilator-induced lung injury (VILI) is characterized by inflammation. The specific mechanism(s) that recruit leukocytes during VILI have not been elucidated. Because the murine CXC chemokines $\mathrm{KC} / \mathrm{CXCL} 1$ and MIP-2/CXCL2/3, via CXCR2, are potent neutrophil chemoattractants, we investigated their role in a murine model of VILI. We compared two ventilator strategies in C57BL/6 mice: high peak pressure and high stretch (high peak pressure/stretch) versus low peak pressure/stretch for 6 hours. Lung injury and neutrophil sequestration from the high-peak pressure/stretch group were greater than those from the low-peak pressure/stretch group. In addition, lung expression of $\mathrm{KC} / \mathrm{CXCL} 1$ and MIP-2/CXCL2/3 paralleled lung injury and neutrophil sequestration. Moreover, in vivo inhibition of CXCR2/CXC chemokine ligand interactions led to a marked reduction in neutrophil sequestration and lung injury. These findings were confirmed using CXCR2 ${ }^{-/-}$mice. Together these experiments support the notion that increased expression of KC/CXCL1 and MIP-2/CXCL2/3 and their interaction with CXCR2 are important in the pathogeneses of VILI.
\end{abstract}

J. Clin. Invest. 110:1703-1716 (2002). doi:10.1172/JCI200215849.

\section{Introduction}

Acute lung injury (ALI) and acute respiratory distress syndrome (ARDS) are clinical syndromes (hypoxemia, decreased lung compliance, and bilateral pulmonary infiltrates) that have multifactorial etiologies either from direct or from indirect injury to the lung $(1,2)$. Histopathologically, there is an initial acute exudative phase involving an alveolar-capillary leak in conjunction with leukocyte extravasation. This is followed by a fibroproliferative phase involving the precipitation of the alveolar proteins with hyaline membrane formation, persist-

Received for publication May 2, 2002, and accepted in revised form August 27, 2002

Address correspondence to: Robert M. Strieter, Department of Medicine, Division of Pulmonary and Critical Care Medicine, and Department of Pathology and Laboratory Medicine, David Geffen School of Medicine at University of California, Los Angeles, Room 14-154 Warren Hall, Box 711922, 900 Veteran Avenue, Los Angeles, California 90024-1922, USA.

Phone: (310) 794-1999; Fax: (310) 794-1998;

E-mail: rstrieter@mednet.ucla.edu.

Conflict of interest: The authors have declared that no conflict of interest exists.

Nonstandard abbreviations used: acute lung injury (ALI); acute respiratory distress syndrome (ARDS); ventilator-induced lung injury (VILI); glutamic acid-leucine-arginine (ELR); myeloperoxidase (MPO); hematoxylin and eosin (H\&E); positive end-expiratory pressure (PEEP). ent inflammation, and proliferation of alveolar epithelia and mesenchymal cells. Finally, there is a fibrotic phase, in which inflammation ends in dysregulated repair with denudation of the basement membrane, excessive matrix deposition, and parenchymal fibrosis $(1,2)$.

Clinically, these patients develop an increased physiological dead space, progressive shunt with hypoxemia, decreased compliance, and pulmonary artery vasculopathy, resulting in high minute ventilation that requires the need for mechanical ventilation (1). Management has consisted of aggressive treatment of the inciting cause, vigilant supportive care while on the ventilator, and the prevention of nosocomial infections. However, the mortality rate from ALI/ARDS is approximately $35-65 \%(1-5)$.

Previously there has been no significant progress made in effective treatment of this disease. However, a recent multi-center randomized controlled trial compared a traditional ventilation strategy (tidal volume of $12 \mathrm{ml} / \mathrm{kg}$ ) with a lung-protective strategy (tidal volume of $6 \mathrm{ml} / \mathrm{kg}$ ). The study included 861 patients and demonstrated that the mortality rate in the lung-protective group was $22 \%$ lower than that in the traditional ventilation group (6). This sentinel study has changed the standard of care for ventilator management of patients with ALI/ARDS. However, it has raised questions about possible mecha- 
nism(s) underlying the decreased mortality. Furthermore, animal models of ventilator-induced lung injury (VILI) have demonstrated that high-peak pressure and highstretch (high-peak pressure/stretch) mechanical ventilation leads to increased neutrophil sequestration and lung injury $(4,7,8)$. Moreover, the specific mediator(s) that orchestrates the recruitment of neutrophils into the lung during VILI, which may perpetuate ALI/ARDS, has not been fully elucidated.

Murine KC/CXCL1 and MIP-2/CXCL2/3 are glutamic acid-leucine-arginine-positive (ELR-positive) CXC chemokines; are structural homologs of human GRO- $\alpha /$ CXCL1 and GRO- $\beta / \gamma /$ CXCL2/3, respectively; and are functional homologs of human CXC chemokines, such as IL-8/CXCL8, ENA-78/CXCL5, and GRO- $\alpha / \beta / \gamma /$ CXCL1/2/3 (9-12). Both murine chemokines share the ability to signal through a $G$ protein-coupled receptor, CXCR2 (9-11). Their human structural and functional homologs have been associated with ALI/ARDS (13-20). Because of these findings, we hypothesized that the lung injury from traditional high-peak pressure/stretch mechanical ventilation leading to a neutrophil-predominant leukocyte infiltration is due, in part, to the expression of ELR-positive CXC chemokines through their interaction with their major receptor, CXCR2.

Our study demonstrates that high-peak pressure/ stretch mechanical ventilation leads to neutrophil sequestration and lung injury that parallel the expression of KC/CXCL1 and MIP-2/CXCL2/3, and of cells expressing CXCR2. Moreover, inhibition of CXCR2/ CXCR2 ligand interactions decreases neutrophil infiltration into the lungs and attenuates VILI.

\section{Methods}

Reagents used for ELISA experiments. Biotinylated and nonbiotinylated anti-murine KC/CXCL1 was purchased from R\&D Systems Inc. (Minneapolis, Minnesota, USA). Polyclonal rabbit anti-murine MIP$2 /$ CXCL2/3 used for ELISA was produced by the immunization of a rabbit with carrier-free murine recombinant MIP-2/CXCL2/3 (R\&D Systems Inc.) in multiple intradermal sites with CFA, followed by at least three boosts of MIP-2/CXCL2/3 in incomplete Freund's adjuvant, as previously described (21-24).

Reagents used for neutralization studies. Polyclonal goat anti-murine CXCR2 was produced by the immunization of a goat with a peptide containing the ligandbinding sequence Met-Gly-Glu-Phe-Lys-Val-Asp-LysPhe-Asn-Ile-Glu-Asp-Phe-Phe-Ser-Gly of CXCR2. The goat was immunized with CXCR2 peptide in a fashion similar to that mentioned above for MIP-2/CXCL2/3 (21-28). Direct ELISA was used to evaluate antiserum titers, and serum was used for Western blot, ELISA, and neutralization assays when titers had reached greater than $1 / 1,000,000$. The CXCR2 protein sequence has been shown to contain the ligand-binding portion of the CXCR2 receptor $(21-23,29)$. The antiCXCR2 antibodies have been used previously to block mouse CXCR2 in vivo and have been shown to detect CXCR2 by Western blot and FACS analysis of neutrophils in vivo $(21-23,29)$. The anti-CXCR2 antibodies have been shown to be neutralizing, both by in vitro neutrophil chemotaxis assay and by in vivo abrogation of the influx of neutrophils into the peritoneum of normal mice in response to exogenous ELR-positive murine CXC chemokines $(21-23,29)$. In vivo administration of anti-CXCR2 antibodies inhibited pulmonary neutrophil sequestration in murine models of Aspergillosis, Nocardia, and Pseudomonas pneumonia and prevented the influx of neutrophils in urine and the kidney in a murine model of Escherichia coli urinary tract infection $(21-23,29)$. Moreover, intraperitoneal administration of this antibody did not alter peripheral blood neutrophil counts $(21-23,29)$. One milliliter of antiserum against murine CXCR2 and control antibody is approximately $10 \mathrm{mg}$ of IgG. Affinity-purified polyclonal anti-KC/CXCL1 and anti-MIP-2/ CXCL2/3 antibodies were purchased from R\&D Systems Inc. Twenty-five micrograms of either anti-KC/ CXCL1 or anti-MIP-2/CXCL2/3 antibodies has the neutralizing capacity to inhibit $1 \mu \mathrm{g} / \mathrm{ml}$ of KC/CXCL1 or MIP-2/CXCL2/3, respectively.

Murine model of VILI. We used a modification of the established and reproducible rodent/rabbit model of VILI in mice as previously described $(7,30,31)$. We used male C57BL/ 6 mice between 6 and 8 weeks old. Mice were anesthetized with ketamine and rompun intraperitoneally; then, under sterile conditions, the anterior neck soft tissue was dissected to expose the trachea, and a tracheotomy was performed. A 22-gauge angiocatheter was introduced into the trachea and sutured in place. Mice were placed in a supine position on a warming device and then connected to a Harvard Apparatus Co. ventilator, model 683 (South Natick, Massachusetts, USA). The high-peak pressure/stretch protocol consisted of maintaining ventilator settings such that the respiratory rate $=100$ breaths per minute and peak pressure $=40 \mathrm{~cm} \mathrm{H} \mathrm{H}_{2} \mathrm{O}$, with tidal volumes of approximately $0.024 \mathrm{ml} / \mathrm{g}$ and an inspired oxygen fraction of 0.21 for 6 hours. The low-peak pressure/stretch protocol consisted of maintaining ventilator settings such that the respiratory rate $=100$ breaths per minute and peak pressure $=20 \mathrm{~cm} \mathrm{H} \mathrm{H}_{2} \mathrm{O}$, with tidal volumes of approximately $0.012 \mathrm{ml} / \mathrm{g}$ and an inspired oxygen fraction of 0.21 for 6 hours. Airway peak pressure was measured with an Exactus II pressure gauge (Omron Health Care Inc., Vernon Hill, Illinois, USA) connected to the tubing at the proximal end of the tracheostomy.

The mice received $1 \mathrm{ml}$ of $0.9 \%$ normal saline 27 and 3 hours prior to being placed on the mechanical ventilator, and then $0.25 \mathrm{ml}$ of normal saline every 2 hours after the onset of mechanical ventilation. While on the ventilator, the mice were monitored every 30 minutes and given ketamine/rompun intraperitoneally as needed for adequate sedation. Mice also had their blood pressure and pulse monitored within the first hour and within the last hour of mechanical ventilation using a 
murine blood pressure/pulse monitor (Visitech Systems, Apex, North Carolina, USA).

Two mice were placed on separate ventilators side by side so that pairs of mice could be studied simultaneously (for high versus low peak pressure/stretch, and for high peak pressure/stretch treated with neutralizing antibodies versus appropriate control antibodies). The mice in the nonventilated control group received a half dose of ketamine/rompun intraperitoneally and had their blood pressure and pulse measured within 1 hour of the injection. In separate experiments, animals received either $25 \mu \mathrm{g}$ of goat anti-murine KC/CXCL1 (R\&D Systems Inc.), $25 \mu \mathrm{g}$ of goat anti-murine MIP-2/CXCL2/3 (R\&D Systems Inc.), $25 \mu \mathrm{g}$ of goat anti-murine KC/CXCL1 plus 25 $\mu \mathrm{g}$ of goat anti-murine MIP-2/CXCL2/3, 1 ml of goat polyclonal anti-murine CXCR2, or appropriate control antibodies 24 and 3 hours before being placed on the ventilator. Furthermore, to substantiate results found using the anti-murine CXCR2 antibody studies, we performed the same studies using CXCR2 $2^{--}$ mice on a C57BL/ 6 background, comparing them with CXCR2 $2^{+/+}$(wild-type) C57BL/6 mice.

Myeloperoxidase assay. Pulmonary neutrophil sequestration was quantitated using a myeloperoxidase (MPO) assay as previously described $(27,32)$. Briefly, at the time of sacrifice, lungs were perfused free of blood with $1 \mathrm{ml}$ of $0.9 \%$ saline via the spontaneously beating right ventricle. The lungs were excised and placed in a $50-\mathrm{mM}$ potassium phosphate buffer solution ( $\mathrm{pH} 6.0$ ) with $5 \%$ hexadecyltrimethyl ammonium bromide (Sigma-Aldrich, St. Louis, Missouri, USA). Thelung tissue was homogenized, sonicated, and centrifuged at $12,000 \mathrm{~g}$ for 15 minutes at $4{ }^{\circ} \mathrm{C}$. The supernatant was then assayed for MPO activity using a spectrophotometric reaction with $o$-dianisidine hydrochloride (Sigma-Aldrich) at $460 \mathrm{~nm}$.

Histopathologic grading of VILI. Three random $5-\mu \mathrm{m}$ paraffin-embedded tissue sections from five different lungs taken from the high-peak pressure/stretch, low-peak pressure/stretch, and normal nonventilated mice were stained with hematoxylin and eosin (H\&E) at 6 hours after ventilation. The histopathology was reviewed in a blinded manner with respect to which group or mouse was being reviewed, using a modified VILI histologic scoring system as previously described $(30,33)$. Four easily identifiable pathologic processes were scored on a scale of $0-4$ as previously described: (a) alveolar congestion, (b) hemorrhage, (c) leukocyte infiltration or aggregation of neutrophils in airspace or the vessel wall, and (d) thickness of the alveolar wall $(30,33)$. A score of 0 represented normal lungs; 1 , mild, $<25 \%$ lung involvement; 2 , moderate, $25-50 \%$ lung involvement; 3 , severe, $50-75 \%$ lung involvement; and 4 , very severe, $>75 \%$ lung involvement. An overall score of VILI was obtained based on the summation of all the scores, and then a mean \pm SEM was generated from the cohort of normal or ventilated lungs (three sections from each lung, five lungs per group) at each time point to generate a cumulative histological VILI.
Microvascular permeability analysis related to lung injury. Microvascular permeability related to lung injury was measured using a modification of the Evans blue dye extravasation technique as previously described (32, 34). Extravasation of Evans blue (Sigma-Aldrich) into the extravascular compartment was used as a quantitative measure of lung injury and changes in pulmonary microvasculature. Briefly, each animal received $20 \mathrm{mg} / \mathrm{kg}$ of Evans blue ( $\mathrm{pH} 7.34$ ) by tail vein injection 3 hours before being taken off the ventilator for sacrifice. At the time of sacrifice, a heparinized sample of blood was harvested, and plasma was removed by centrifugation. The lungs were perfused free of blood with $1 \mathrm{ml}$ of $0.9 \%$ normal saline via the spontaneously beating right ventricle and removed from the thoracic cavity. The trachea, mainstem bronchi, and surrounding mediastinal structures were removed. Evans blue was extracted from pulmonary tissues after homogenization in $1 \mathrm{ml}$ of $0.9 \%$ normal saline. This volume was added to 2 volumes of deionized formamide and incubated at $60^{\circ} \mathrm{C}$ for 12 hours. The supernatant was separated by centrifugation at 2,000 $\mathrm{g}$ for 30 minutes. Evans blue in the plasma and lung tissue was quantitated by dual-wavelength spectrophotometric analysis at 620 and $740 \mathrm{~nm}$ (35). This method corrects the specimen's absorbance at $620 \mathrm{~nm}$ for the absorbance of contaminating heme pigments, using the following formula: corrected absorbance at $620 \mathrm{~nm}=$ actual absorbance at $620 \mathrm{~nm}-[1.426($ absorbance at 740) + 0.03]. We calculated a permeability index by dividing the corrected pulmonary tissue Evans blue absorbance at $620 \mathrm{~nm} / \mathrm{g}$ of lung tissue by the corrected plasma Evans blue absorbance at $620 \mathrm{~nm}$; this index reflects the degree of extravasation of Evans blue into the extravascular pulmonary tissue compartment.

To quantitate lung edema during VILI, we obtained wet-to-dry weight ratios by ligating the lungs away from the hilum. The lungs were blotted dry and weighed. They were then desiccated by incubation at $130^{\circ} \mathrm{C}$ overnight in a vacuum oven and re-weighed to determine their dry weight. The wet-to-dry ratio was then calculated.

KC/CXCL1 and MIP-2/CXCL2/3 ELISAs. KC/CXCL1 or MIP-2/CXCL2/3 protein was quantitated using a modification of a double ligand method as previously described $(27,28)$. Briefly, flat-bottomed 96-well microtiter plates (Nunc-Immuno-Plate I 96-F, Nalge Nunc International, Rochester, New York, USA) were coated with anti-murine KC/CXCL1 or MIP-2/ CXCL2 $/ 3\left(1 \mathrm{ng} / \mu \mathrm{l}\right.$ in $0.6 \mathrm{M} \mathrm{NaCl}, 0.26 \mathrm{M} \mathrm{H}_{3} \mathrm{BO}_{4}$, and $0.08 \mathrm{~N} \mathrm{NaOH}, \mathrm{pH} 9.6$ ) for 24 hours at $4^{\circ} \mathrm{C}$ and then washed with PBS (pH 7.5) and 0.05\% Tween-20 (wash buffer). Microtiter plate nonspecific binding sites were blocked with $2 \%$ BSA in PBS and incubated for $60 \mathrm{~min}$ utes at $37^{\circ} \mathrm{C}$. Plates were washed three times with wash buffer, and samples or standard were added, followed by incubation for 1 hour at $37^{\circ} \mathrm{C}$. Plates were washed three times, $50 \mu \mathrm{l} /$ well of biotinylated anti-murine KC/CXCL1 and MIP-2/CXCL2/3 antibodies were added, and plates were incubated for 45 minutes at 


\section{Table 1}

Hemodynamic monitoring of normal nonventilated mice and mice placed on either a high- or a low-peak pressure/stretch mechanical ventilation protocol for 6 hours

\begin{tabular}{|c|c|c|c|c|c|c|c|c|c|}
\hline \multirow{2}{*}{\multicolumn{2}{|c|}{ Normal nonventilated C57BL/ }} & \multicolumn{4}{|c|}{ Low peak pressure/stretch } & \multicolumn{4}{|c|}{ High peak pressure/stretch } \\
\hline & & \multicolumn{2}{|c|}{ First hour } & \multicolumn{2}{|c|}{ Sixth hour } & \multicolumn{2}{|c|}{ First hour } & \multicolumn{2}{|c|}{ Sixth hour } \\
\hline $\mathrm{BP}$ & Pulse & $\mathrm{BP}$ & Pulse & $\mathrm{BP}$ & Pulse & $\mathrm{BP}$ & Pulse & $\mathrm{BP}$ & Pulse \\
\hline $103 \pm 8$ & $485 \pm 15$ & $109 \pm 14$ & $485 \pm 19$ & $94 \pm 6$ & $505 \pm 8$ & $97 \pm 6$ & $499 \pm 16$ & $89 \pm 7$ & $511 \pm 14$ \\
\hline
\end{tabular}

$n=10$ mice per group. BP, systolic blood pressure.

$37^{\circ} \mathrm{C}$. Plates were washed three times, streptavidin-peroxidase conjugate (Jackson ImmunoResearch Laboratories Inc., West Grove, Pennsylvania, USA) was added, and the plates were incubated for 30 minutes at $37^{\circ} \mathrm{C}$. Plates were washed three times, and TMB $\left(3,3^{\prime}, 5,5^{\prime}\right.$ tetramethylbenzidine) chromogen substrate (Kirkegaard \& Perry Laboratories Inc., Gaithersburg, Maryland, USA) was added. The plates were incubated at room temperature to the desired extinction, and the reaction was terminated with $3 \mathrm{M} \mathrm{H}_{2} \mathrm{SO}_{4}$ solution. Plates were read at $450 \mathrm{~nm}$ in an automated microplate reader (Bio-Tek Instruments Inc., Winooski, Vermont, USA). Standards were half-log dilutions of either KC/CXCL1 or MIP-2/CXCL2/3 from $100 \mathrm{ng}$ to $1 \mathrm{pg} / \mathrm{ml}$ $(50 \mu \mathrm{l} /$ well). This ELISA method consistently detected specific chemokine concentrations greater than 50 $\mathrm{pg} / \mathrm{ml}$ in a linear fashion. KC/CXCL1 and MIP-2/ CXCL2 $/ 3$ were specific in our sandwich ELISA without cross-reactivity to a panel of cytokines including human and murine IL-1Ra, IL-1, IL-2, IL-6, IL-4, TNF- $\alpha$, IFN- $\gamma$, and members of the CXC and CC chemokine families.

ELISA for phosphorylated I $\mathrm{I} B \alpha$ and Western blot analysis of $I \kappa B \alpha$. To assess activated NF- $\kappa B$ in tissue, phosphorylated I $\kappa \alpha \alpha$ from whole-lung homogenates was measured using a modification of a commercially available ELISA kit, IкB $\alpha$ ActivELISA (BioCarta, San Diego, California, USA). Briefly, a flat-bottomed 96-well microtiter plate was coated with capture antibody for 24 hours at $4^{\circ} \mathrm{C}$ and then washed. Nonspecific binding sites were blocked with blocking buffer (BioCarta) and incubated for 60 minutes at room temperature. The plate was washed three times with wash buffer (BioCarta), and samples or standard were added, followed by incubation for 4 hours at $4^{\circ} \mathrm{C}$. The plate was washed three times with wash buffer (BioCarta), and detection antibody was added, followed by incubation for 1 hour at room temperature. The plate was further washed three times, and streptavidin-horseradish peroxidase added. The plate was then incubated for 1 additional hour at room temperature. The plate was washed three times with wash buffer (BioCarta), and TMB chromogen substrate (Kirkegaard \& Perry Laboratories Inc.) was added. The plate was incubated at room temperature to the desired extinction, and the reaction was terminated with $3 \mathrm{M} \mathrm{H}_{2} \mathrm{SO}_{4}$ solution. The plate was read at $450 \mathrm{~nm}$ in an automated microplate reader (Bio-Tek Instruments Inc.). The standard curve was generated using half dilutions of phosphorylated IKB $\alpha$ from $1,000 \mu \mathrm{g} / \mathrm{ml}$ to 0 , provided by the manufacturer (BioCarta).

We performed Western blot analysis by immunoblotting $60 \mu \mathrm{g}$ of total protein from whole-lung homogenate preparations. Following SDS-PAGE, lung homogenate protein was electrophoretically transferred to a PVDF membrane at $30 \mathrm{~V}$ for 1 hour at room temperature. The membranes were then incubated with an anti-I $\kappa \mathrm{B} \alpha$ antibody (Santa Cruz Biotechnology Inc., Santa Cruz, California, USA) at a 1:1,000 dilution in BLOTTO (Cell Signaling Technology, Beverly, Massachusetts, USA) overnight at $4^{\circ} \mathrm{C}$. Subsequently, the blots were washed in Tween 20-Tris buffered saline and then incubated with a horseradish peroxidase-conjugated donkey anti-rabbit secondary antibody for 1 hour at room temperature. The immunoreactive proteins were finally visualized using ECL (Amersham Biosciences Corp., Piscataway, New Jersey, USA) and by following the manufacturer's instructions. Bands were visualized and photographed using an ultraviolet transilluminator (36). The image densities were measured with NIH Image, and semiquantitative results were obtained.

Total-RNA isolation and RT-PCR amplification. Total cellular RNA from tracheal tissue was isolated as previously described (25-28). Total RNA was determined, and $1 \mu \mathrm{g}$ of total RNA was reverse-transcribed into cDNA and amplified using Access RT-PCR kit (Promega Corp., Madison, Wisconsin, USA) and specific primers for murine KC/CXCL1, murine MIP2/CXCL2/3, murine CXCR2, and a housekeeping gene, $\beta$-actin. The primers used were $5^{\prime}$-TGA-GCT-GCGCTG-TCA-GTG-CCT-3' (sense) and 5'-AGA-AGC-CAGCGT-TCA-CCA-GA-3' (antisense) for KC (259 bp); $5^{\prime}$-GCT-GGC-CAC-CAA-CCA-CCA-GG-3' (sense) and 5'AGC-GAG-GCA-CAT-CAG-GTA-CG-3' (antisense) for murine MIP-2/CXCL2/3 (359 bp); 5'-TCT-GGC-ATGCCC-TCT-ATT-CTG-3' (sense) and 5'-AAG-GTA-ACC-TCCTTC-ACG-TAT-3' (antisense) for murine CXCR2 (311 bp); and 5'-TTG-TAA-CCA-ACT-GGG-ACG-ATA-TGG-3' (sense) and 5'-GAT-CTT-GAT-CTT-CAT-GGT-GCT-AGG-3' (antisense) for $\beta$-actin (764 bp). After amplification, the sample was separated on an agarose gel containing ethidium bromide, and bands were visualized and photographed using an ultraviolet transilluminator (36). The image densities were measured with NIH Image, and semiquantitative results were expressed as a ratio of each RT-PCR product to $\beta$-actin density. 

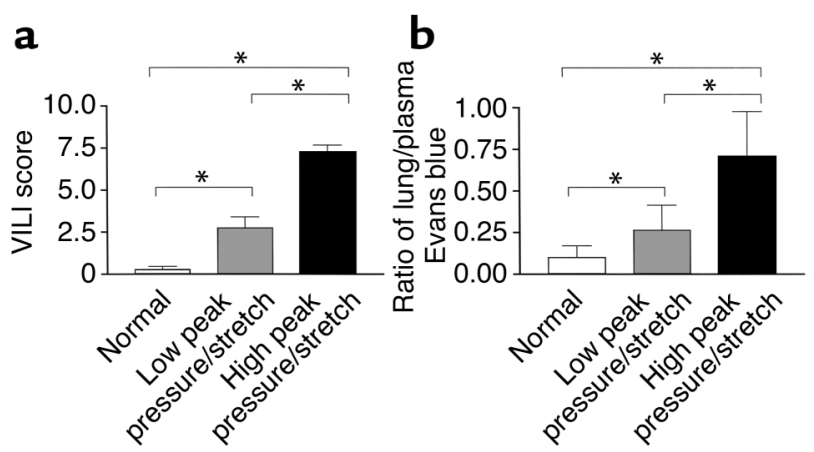

\section{Figure 1}

VILI. (a) VILI scores by analysis of H\&E-stained histopathologic sections from mouse lungs after 6 hours of mechanical ventilation. A cumulative score was based on leukocyte infiltration, exudative edema, hemorrhage, and alveolar wall thickness ( $n=15$; three random sections per lung and five lungs per group). ${ }^{*} P<0.02$. (b) Evans blue permeability index where Evans blue was given 3 hours after the start of mechanical ventilation and mice were sacrificed at the 6-hour time point ( $n=10$ lungs per group). ${ }^{*} P<0.02$.

FACS analysis of CXCR2. Whole-lung single-cell suspension preparations were made from harvested lungs using a method described previously $(28,37,38)$. Singlecell suspensions $\left(5 \times 10^{6} \mathrm{cell} / \mathrm{s} / \mathrm{ml}\right)$ were stained with primary antibodies: Tricolor-conjugated (Becton, Dickinson and Company, Franklin Lakes, New Jersey, USA) anti-murine CD45 (Caltag Laboratories Inc., South San Francisco, California, USA), anti-murine CXCR2, or isotype controls. The secondary antibody used for these experiments was Alexa 488 (Molecular Probes Inc., Eugene, Oregon, USA). Dual-color stained cell suspensions were analyzed on a FACScan flow cytometer (Becton Dickinson Immunocytometry Systems, San Jose, California, USA) using CellQuest 3.2.1f1 software (Becton Dickinson Immunocytometry Systems) as previously described $(28,37,38)$.

Statistical analysis. Data were analyzed on a Dell PC using the StatView 4.5 statistical package (Abacus Concepts Inc., Berkeley, California, USA). Group comparisons were evaluated by the ANOVA test with the post hoc analysis (i.e., Bonferroni/Dunn). Data were expressed as mean \pm SEM.

\section{Results}

High-peak pressure/stretch mechanical ventilation is associated with increased VILI. Since human studies have suggested that high-peak pressure/stretch mechanical ventilation is associated with increased lung injury, we generated a murine model system of high- versus low-peak pressure/stretch mechanical ventilation in order to begin to dissect the mechanism(s) related to this process. C57BL/6 mice were placed on either a high- or a low-peak pressure/stretch protocol of mechanical ventilation at a respiratory rate of 100 for 6 hours. Hemodynamic monitoring did not demonstrate any significant difference in blood pressure or pulse, in either group, at the beginning versus the end of mechanical ventilation (Table 1). By performing quantitative analysis on lung histopathology, we found more edema, leukocyte infiltration, alveolar hemorrhage, and alveolar wall thickness in lungs harvested from the high-peak pressure/stretch mice (high peak pressure/stretch > > low peak pressure/stretch > nonventilated normal mice) (Figures $1 \mathrm{a}$ and 2 ). We confirmed these results by performing Evans blue dye analysis, a marker of microvascular permeability. Evans blue dye was infused into mice by tail vein injection 3 hours after the start of mechanical ventilation. At 6 hours after ventilation, the animals were sacrificed and the lungs and serum were harvested for microvascular permeability index analysis. We found that lungs from the high-peak pressure/stretch group had a significantly higher vascular permeability than those from either the low-peak pressure/stretch group or normal nonventilated mice (Figure $1 \mathrm{~b}$ ). In addition, there was an increase in vascular permeability in lungs from the low-peak pressure/stretch group as compared with normal nonventilated mice (Figure $1 \mathrm{~b}$ ).

MPO levels are elevated during VILI. Neutrophils are considered to be critically involved in the pathogenesis of most forms of ALI and are one of the histopathological hallmarks of ALI/ARDS $(1,39-44)$. Based on this evidence, we measured neutrophil sequestration in the lung during both high- and low-peak pressure/stretch mechanical ventilation, as compared with that of normal non-ventilated control mice. C57BL/6 mice placed on either a high- or a low-peak pressure/stretch mechanical ventilation were sacrificed at 6 hours, and their lungs were harvested for MPO analysis. MPO was used as an indirect measurement of neutrophil infiltration into the lung. Lung homogenates from the high-peak pressure/stretch group had significantly greater levels of MPO than lung homogenates from either low-peak pressure/stretch or normal nonventilated mice (Figure 3). Furthermore, MPO levels were higher in the lungs of the low-peak pressure/stretch

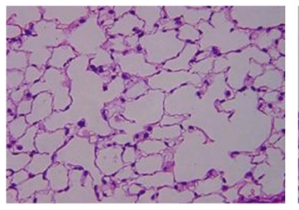

Normal

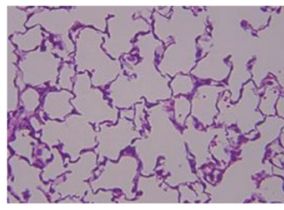

Low peak pressure/stretch

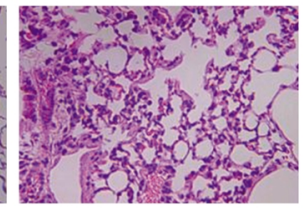

High peak pressure/stretch
Six hours after mechanical ventilation

\section{Figure 2}

Histopathology of lungs is associated with mechanical ventilation. Representative photomicrographs with H\&E staining $(\times 400)$ of the lung histopathology from mice placed on either high- or low-peak pressure/stretch mechanical ventilation, or from normal nonventilated mice. 


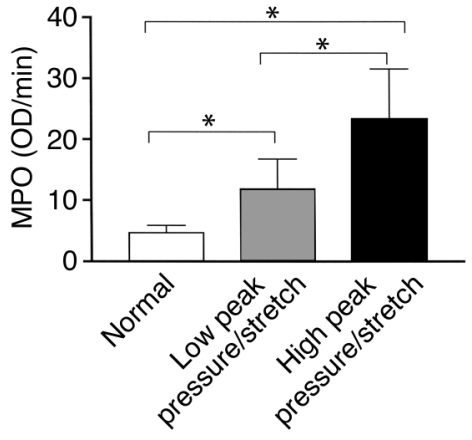

Figure 3

Lung neutrophil sequestration as demonstrated by MPO levels from mice placed on either high- or low-peak pressure/stretch mechanical ventilation, or from normal nonventilated mice $(n=10$ mice per group). ${ }^{*} P<0.02$.

group than in the lungs of normal nonventilated mice (Figure 3). The findings of elevated MPO in the lungs of the high-peak pressure/stretch group suggest that neutrophil sequestration is associated with lung injury.

$K C / C X C L 1$ and MIP-2/CXCL2/3 mRNA expression and protein levels are elevated during VILI. Finding increased levels of MPO that paralleled VILI led us to hypothesize that chemotactic factors for neutrophils were expressed and mediate the recruitment of these cells. On this basis we evaluated the expression of two potent CXC chemokine neutrophil chemoattractants, KC/CXCL1 and MIP-2/CXCL2/3. Lung homogenates were analyzed at 6 hours after ventilation from mice on high- or low-peak pressure/stretch mechanical ventilation, compared with normal nonventilated mice. We found significantly higher levels of mRNA expression of KC/CXCL1 and MIP-2/CXCL2/3 in the lungs from the high-peak pressure/stretch ventilated group, as compared with either the low-peak pressure/stretch or the normal nonventilated lungs (Figure 4 , $a$ and $b$ ). In addition, there was greater mRNA expression of KC/CXCL1 and MIP-2/CXCL2/3 in the low-peak pressure/stretch group than in the normal nonventilated group (Figure 4, a and b). Similarly, both KC/CXCL1 and MIP-2/ CXCL2 $/ 3$ protein levels in the lungs were greatest in the high-peak pressure/stretch group (Figure 5, a and b). These findings suggest that the expression of KC/CXCL1 and MIP-2/CXCL2/3 paralleled the neutrophil sequestration and VILI (Figures 1-5). The expression of these two CXC chemokines was compartmentalized to the lungs, as serum levels for these two chemokines were found to be similar for all three groups of mice (Figure 5, b and c).

$N F-\kappa B$ is activated and correlates with expression of $K C / C X C L 1$ and MIP-2/CXCL2/3. NF- $\mathrm{KB}$ is a critical transcription factor for the expression of KC/CXCL1 and MIP-2/CXCL2/3 during inflammation. Phosphorylation of I $\mathrm{I} B \alpha$ leads to ubiquitination, unmasking the nuclear localization signal of NF- $\kappa B$ dimer, and to subsequent translocation to the nucleus and activation of target genes such as KC/CXCL1 and MIP-2/CXCL2/3. The analysis of the phosphorylation of IKB $\alpha$ correlates a

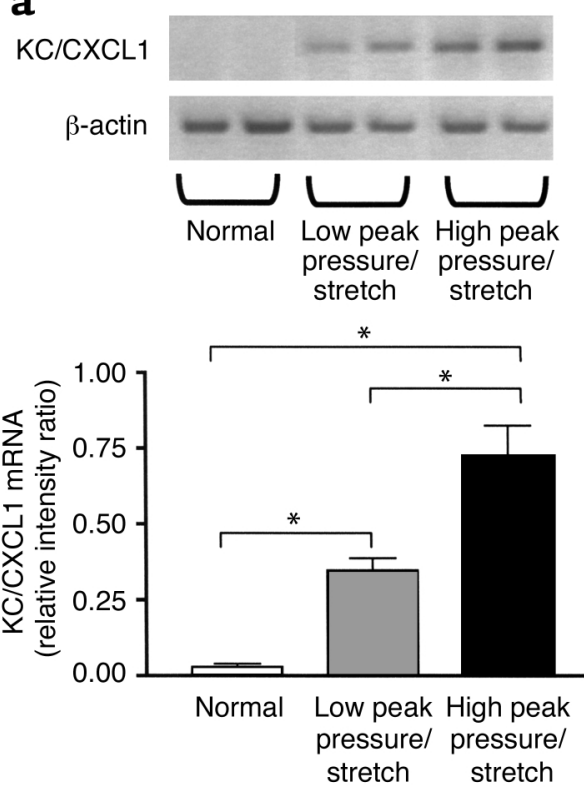

b

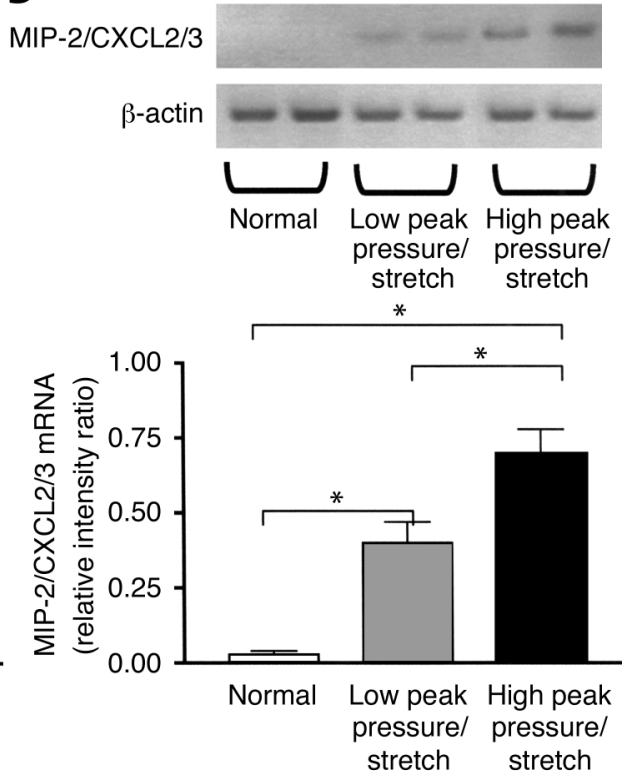

\section{Figure 4}

KC/CXCL1 and MIP-2/CXCL2/3 mRNA levels are markedly elevated during VILI. (a) RT-PCR determination of KC/CXCL1 mRNA in lung homogenates from mice placed on either high- or low-peak pressure/stretch ventilator protocol, or from normal nonventilated mice. Semiquantitative results are expressed as a ratio of each PCR product to $\beta$-actin band density $\left(n=4\right.$ mice per group). ${ }^{*} P<0.02$. (b) RT-PCR determination of MIP-2/CXCL2/3 mRNA in lung homogenates from mice placed on either high- or low-peak pressure/stretch ventilator protocol, or from normal nonventilated mice. Semiquantitative results are expressed as a ratio of each PCR product to $\beta$-actin band density $\left(n=4\right.$ mice per group). ${ }^{*} P<0.02$. 


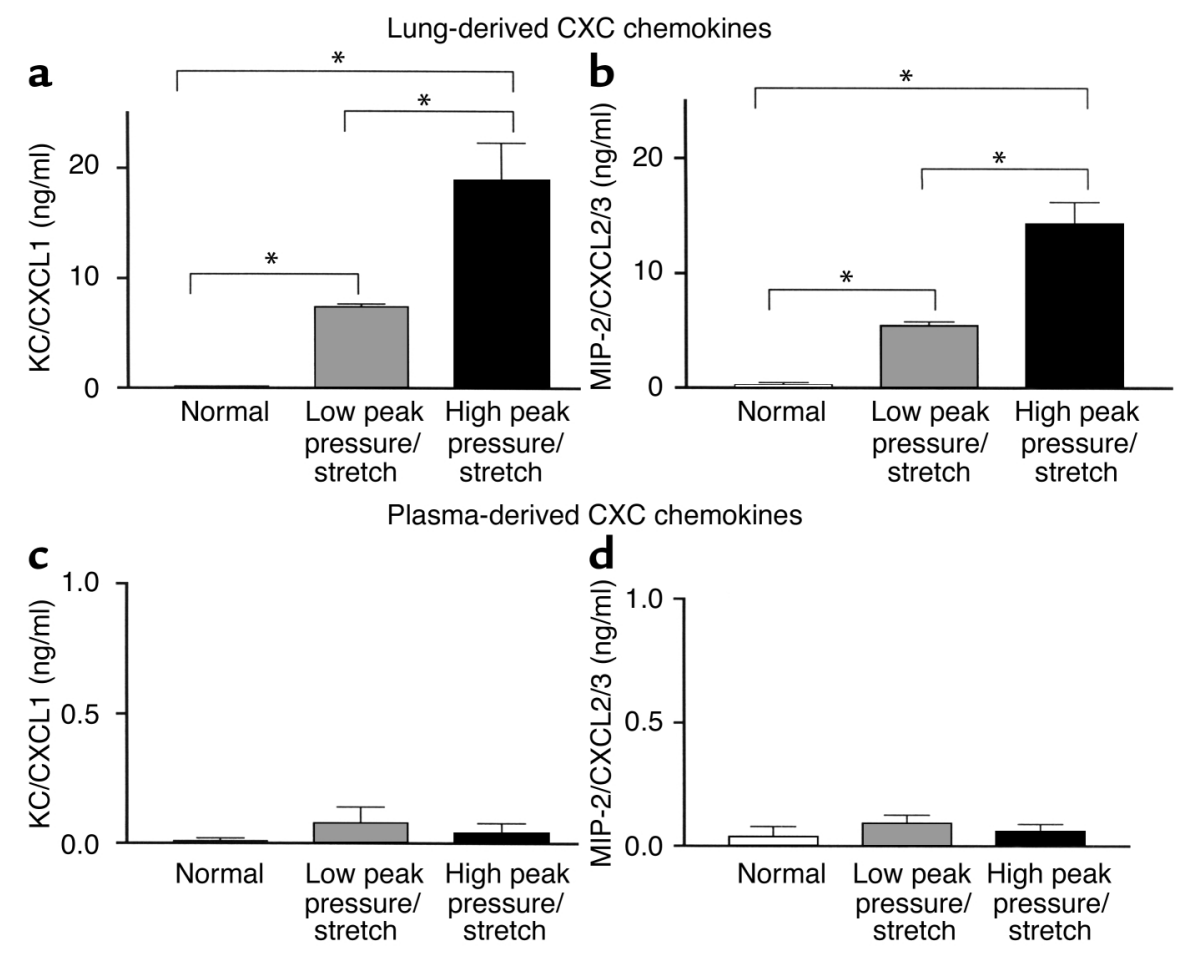

\section{Figure 5}

KC/CXCL1 and MIP-2/CXCL2/3 protein levels are markedly elevated during VILI. ( $\mathbf{a}$ and $\mathbf{b}$ ) ELISA measurements of (a) $\mathrm{KC} / \mathrm{CXCL} 1$ protein levels and (b) MIP-2/ $\mathrm{CXCL2/3}$ protein levels in lung homogenates from mice placed on either high- or low-peak pressure/stretch ventilator protocol, or from normal nonventilated mice ( $n=10$ mice per group). ${ }^{*} P<0.02$. (c and d) ELISA measurements of (c) KC/CXCL1 protein levels and (d) MIP-2/CXCL2/3 protein levels in plasma from mice placed on either highor low-peak pressure/stretch ventilator protocol, or from normal nonventilated mice $(n=10$ mice per group). $P<0.02$. with nuclear localization and activation of NF- $\mathrm{KB}$ (45-47). Lung homogenates were analyzed at 2 hours after ventilation from mice on high- or low-peak pressure/stretch mechanical ventilation, as compared with normal nonventilated mice. We found significantly higher levels of phosphorylated I $\mathrm{I} B \alpha$ in the lungs from the high-peak pressure/stretch ventilated group than in the normal nonventilated lungs (Figure 6a). In addition, there were increased levels of phosphorylated I $\mathrm{KB} \alpha$ in the lungs from the low-peak pressure/stretch group, as compared with the normal nonventilated lungs (Figure 6a). To further confirm activation of NF-KB, Western blot analysis was performed for IKB $\alpha$ protein to assess degradation related to ubiquitination.
We found significantly lower levels of I $\mathrm{I} B \alpha$ protein in the lungs from the high-peak pressure/stretch ventilated group than in the normal nonventilated lungs (Figure 6b). Similarly, there were significantly lower levels of IKB $\alpha$ protein in the lungs from the low-peak pressure/stretch ventilated group than in the normal nonventilated lungs (Figure $6 \mathrm{~b}$ ).

CXCR2 expression is increased during VILI. CXCR2 is the shared cellular receptor for the murine $\mathrm{CXC}$ chemokine ligands KC/CXCL1 and MIP-2/CXCL2/3 (9-11). The finding of increased levels of KC/CXCL1 and MIP-2/ CXCL2/3 associated with VILI and neutrophil sequestration led us to evaluate the expression of CXCR2 in the lungs of these animals. We found markedly greater

\section{Figure 6}

There is increased phosphorylation of $\mathrm{I} \mathrm{\kappa} \mathrm{B} \alpha$ and more degradation of $\mid \kappa \mathrm{B} \alpha$ during the pathogenesis of VILI. (a) Measurements of phosphorylation state of $I \kappa B \alpha$ in whole-lung homogenates from mice placed on either high- or low-peak pressure/stretch ventilator protocol for 2 hours, or from normal nonventilated mice $\left(n=6\right.$ mice per group). ${ }^{*} P<0.02$. (b) Western blot analysis of $\mathrm{I} \kappa \mathrm{B} \alpha$ protein levels in whole-lung homogenates from mice placed on either high- or low-peak pressure/stretch ventilator protocol for 2 hours, or from normal nonventilated mice. Semiquantitative results are expressed as a band density area $(n=6$ mice per group). ${ }^{*} P<0.02$.
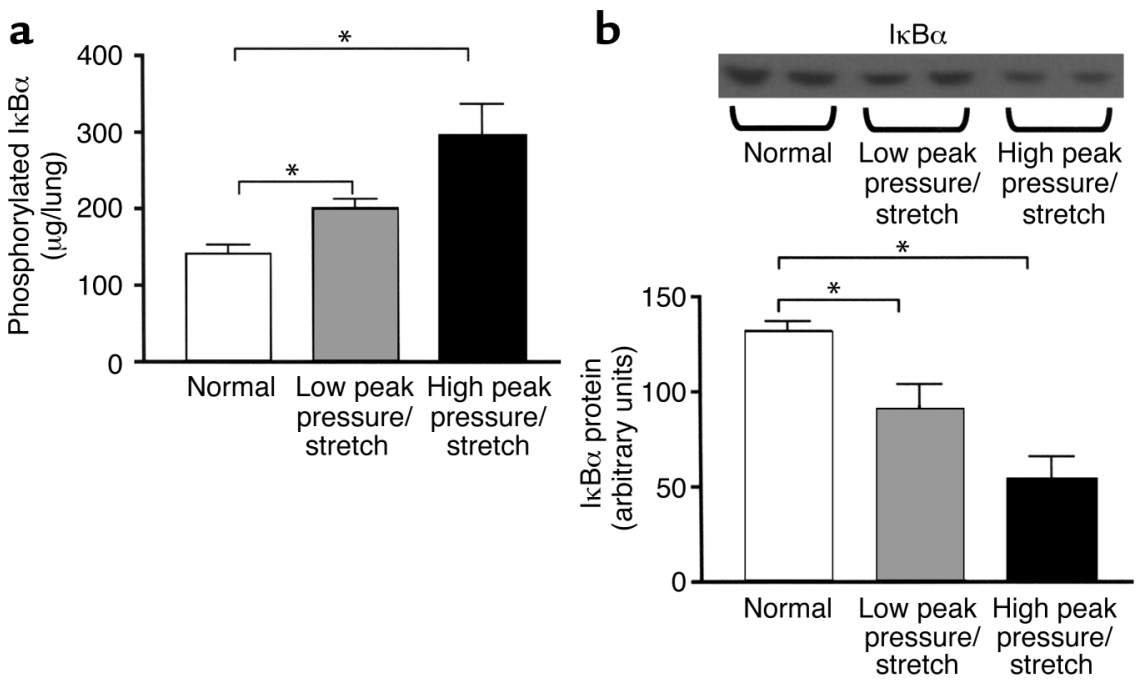
a
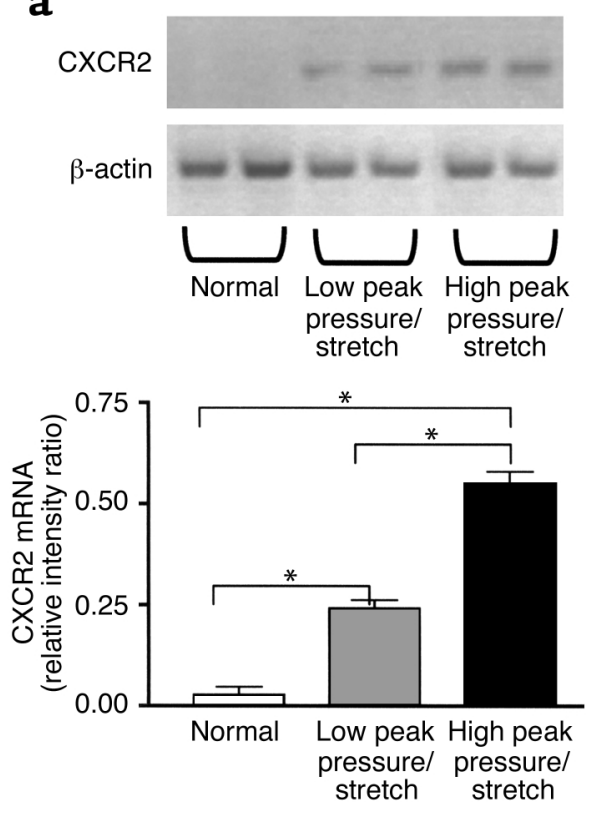
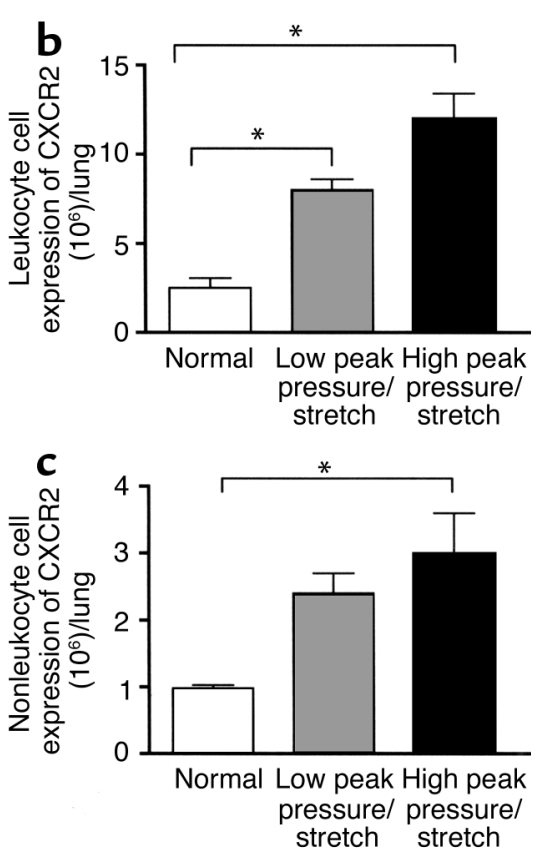

Figure 7

(a) CXCR2 mRNA expression is markedly elevated during VILI. RT-PCR determination of CXCR2 mRNA in lung homogenates from mice placed on either high- or low-peak pressure/ stretch ventilator protocol, or from normal nonventilated mice. Semiquantitative results are expressed as a ratio of each PCR product to $\beta$-actin band density ( $n=4$ mice per group). ${ }^{*} P<0.02$. (b and $\mathbf{c}$ ) FACS analysis of CXCR2. (b) Total number of leukocytes (CD45-positive cells) with cell surface expression of CXCR2 in whole-lung digest homogenates from mice placed on either high- or low-peak pressure/ stretch ventilator protocol, or from normal nonventilated mice ( $n=3$ mice per group). ${ }^{*} P<0.02$. (c) Total number of nonleukocytes (CD45-negative cells) with cell surface expression of CXCR2 in whole-lung digest homogenates from mice placed on either high- or low-peak pressure/stretch ventilator protocol, or from normal nonventilated mice $(n=3$ mice per group). ${ }^{*} P<0.02$.
mRNA expression of CXCR2 in lung homogenates from the high-peak pressure/stretch group as compared with either the low-peak pressure/stretch group or normal nonventilated mice (Figure $7 \mathrm{a}$ ). In addition, there were greater levels of mRNA expression of CXCR2 in the lungs from the low-peak pressure/stretch group, as compared with the normal nonventilated mice (Figure 7a). The expression of CXCR2 paralleled KC/CXCL1 and MIP-2/CXCL2/3 expression, neutrophil sequestration, and VILI (Figures 1-7). We confirmed these findings by performing dual-color FACS analysis on singlecell suspensions of whole-lung digests for the cell surface expression of CD45 and CXCR2 protein. There were significant increases in cell surface expression of CXCR2 from both the CD45-positive (leukocyte) and the CD45-negative (nonleukocyte) populations in lung digests from the high-peak pressure/stretch group, as compared with normal nonventilated mice (Figure 7, b and c). In addition, there were greater levels of cell surface expression of CXCR2 from the CD45-positive cell populations in lung digests from the low-peak pressure/stretch group, as compared with normal nonventilated mice (Figure 7, b and c).

Inbibition of KC/CXCL1 and MIP-2/CXCL2/3 attenuates VILI. Having found that the expression of CXCR 2 and its ligands KC/CXCL1 and MIP-2/CXCL2/3 paralleled VILI and neutrophil sequestration, we next determined whether inhibition of specific ligand/CXCR2 biology could attenuate VILI. Mice were passively immunized with specific neutralizing anti-murine KC/CXCL1 antibodies or with control antibodies at 24 and 3 hours prior to being placed on the high-peak pressure/ stretch mechanical ventilation protocol for 6 hours. We found that the lungs from the high-peak pressure/ stretch group treated with neutralizing antibodies to $\mathrm{KC} / \mathrm{CXCL} 1$ had significantly lower levels of VILI than
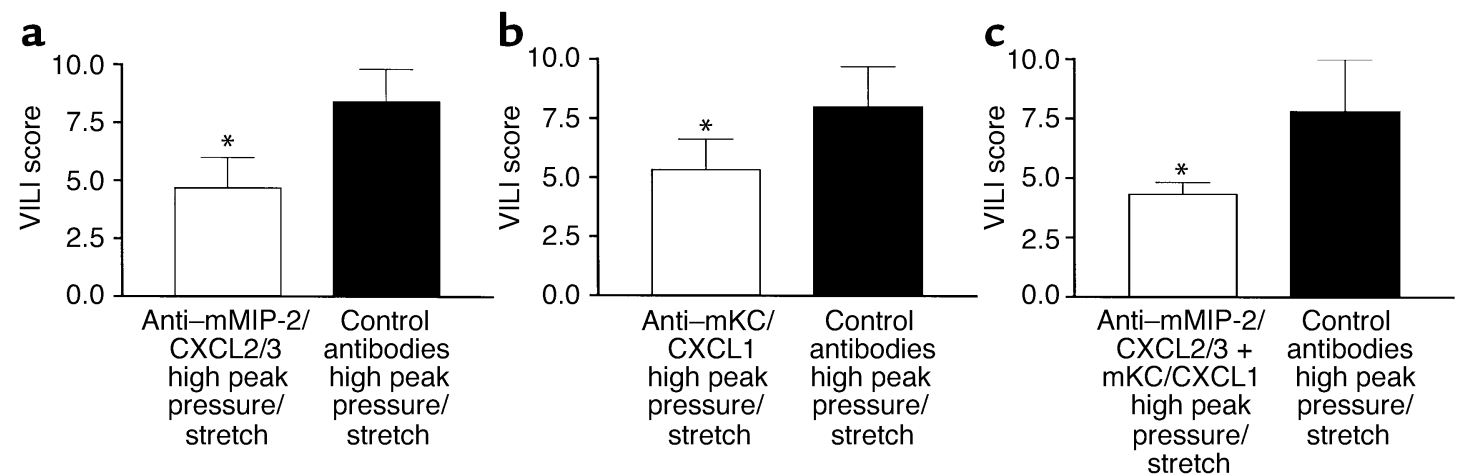

Figure 8

In vivo neutralization of (a) mMIP-2/CXCL2/3, (b) mKC/CXCL1, and (c) mKC/CXCL1 + mMIP-2/CXCL2/3 (as compared with treatment with control antibodies) attenuates VILI, as demonstrated by VILI scores of H\&E-stained histopathologic sections from mice placed on high peak pressure/stretch. A cumulative score was based on leukocyte infiltration, exudative edema, hemorrhage, and alveolar wall thickness ( $n=6$; two random sections per lung and three lungs per group). ${ }^{*} P<0.05$. 


\section{Table 2}

Hemodynamic monitoring of normal nonventilated mice given control antibody, and mice placed on a high-peak pressure/stretch mechanical ventilation protocol for 6 hours with neutralizing antibodies to murine CXCR2 or with control antibodies

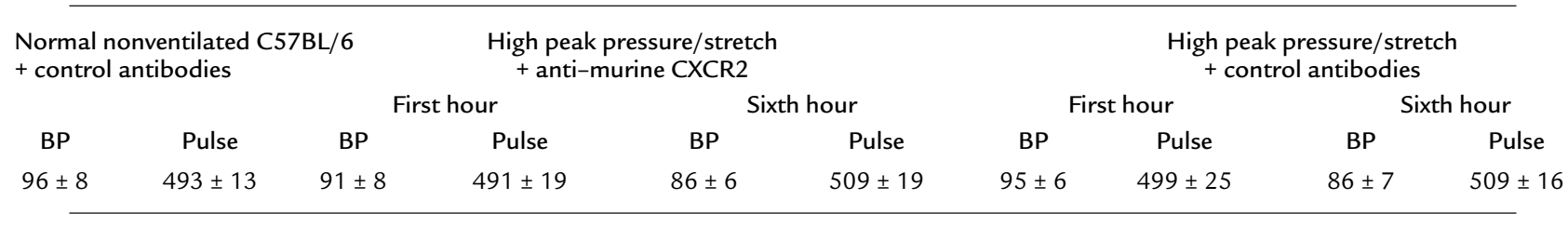

$n=10$ mice per group. BP, systolic blood pressure.

the lungs from the high-peak pressure/stretch group treated with control antibodies (Figure 8b). Similar results were found when mice were passively immunized with either neutralizing anti-murine MIP-2/ $\mathrm{CXCL} 2 / 3$, or the combination of anti-murine $\mathrm{KC} /$ CXCL1 and anti-murine MIP antibodies (Figure 8, a and c, respectively).

Inbibition of CXCR2 inbibits infiltration of neutrophils and attenuates VILI. To better understand the mechanism partly responsible for VILI, we determined whether inhibiting multiple CXCR2 chemokine ligand interactions with CXCR2 significantly decreased neutrophil recruitment to the lung during the pathogenesis of VILI. Mice were passively immunized with specific neutralizing anti-murine CXCR2 or with control antibodies at 24 and 3 hours prior to being placed on the high-peak pressure/stretch mechanical ventilation protocol for 6 hours. Hemodynamic monitoring demonstrated no significant difference in blood pressure or pulse at the beginning versus the end of mechanical ventilation (Table 2). We found that the lungs from the high-peak pressure/stretch group treated with neutralizing antibodies to CXCR2 had significantly lower levels of MPO than the lungs from the high-peak pressure/stretch group treated with control antibodies (Figure 9). Furthermore, inhibiting CXCR2/CXCR2 ligand interactions attenuated VILI, as demonstrated by a reduction in both quantitative histopathologic VILI scores and vascular permeability (Figure 10, a and b; and Figure 11). Moreover, these results were confirmed by a genetic approach in which CXCR2-/- mice were placed on the same mechanical ventilation protocol and compared with $\mathrm{CXCR}^{+/+}$control mice. Hemodynamic monitoring demonstrated no significant difference in either blood pressure or pulse at the beginning and end of mechanical ventilation in either group (Table 3). CXCR2 ${ }^{-/}$mice exposed to high peak pressure/stretch were found to have both markedly reduced and histopathologic VILI scores and lung edema as determined by wet-to-dry weight ratio, as compared with $\mathrm{CXCR2}^{+/+}$mice (Figure 10, $\mathrm{c}$ and d; and Figure 11).

\section{Discussion}

ALI and ARDS are characterized by an initial microvascular leak with a neutrophil-predominant inflammatory response, which promotes diffuse alveolar damage $(1,48,49)$. Mechanical ventilation, while necessary to support patients with ALI or ARDS, has been implicat- ed as a mediator of lung injury (i.e., VILI) during ALI and ARDS (50). In fact, more recent studies have demonstrated that patients with ALI or ARDS who were ventilated with a lung-protective strategy (i.e., low peak pressure/stretch and tidal volume) had a reduced mortality (51-53). Like early ALI and ARDS, VILI is characterized by noncardiogenic edema, neutrophil sequestration, alveolar hemorrhage, and increased alveolar wall thickness $(7,31,54)$. Traditional high-peak pressure/stretch mechanical ventilation may cause overdistension and shearing of the alveolar units, leading to deformation and injury of the cellular components of the alveolar-capillary wall, and thus the release of inflammatory mediators and chemokines. The generation of specific neutrophil chemotactic proteins promotes neutrophil recruitment to the alveolar-interstitial space. This leads to an overexuberant injury and promotes dysregulated repair, leading to perpetuation of ALI/ARDS. In this study, we hypothesized that ELRpositive CXC chemokines expressed during high-peak pressure/stretch mechanical ventilation mediate neutrophil recruitment, a pivotal process required for VILI.

To determine whether CXCR2/CXCR2 ligand interaction contributes to the pathogenesis of VILI, we performed studies using a murine model. We initially characterized this model by determining whether lung injury was dependent on the magnitude of peak pressure/stretch during ventilation. A recent human study using the ARDSNet low-peak pressure/stretch protocol

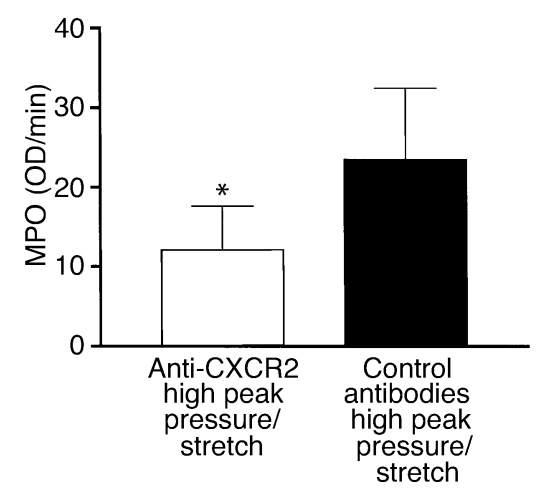

\section{Figure 9}

In vivo neutralization of murine CXCR2 (as compared with treatment with control antibodies) attenuates lung neutrophil sequestration as determined by MPO levels from mice placed on a high-peak pressure/stretch ventilator protocol $\left(n=10\right.$ mice per group). ${ }^{*} P<0.05$. 
a

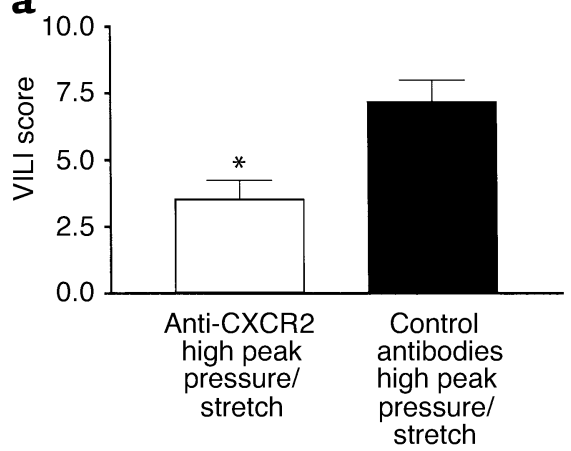

c

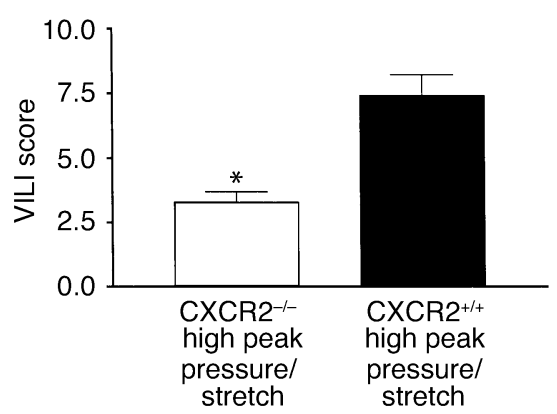

b

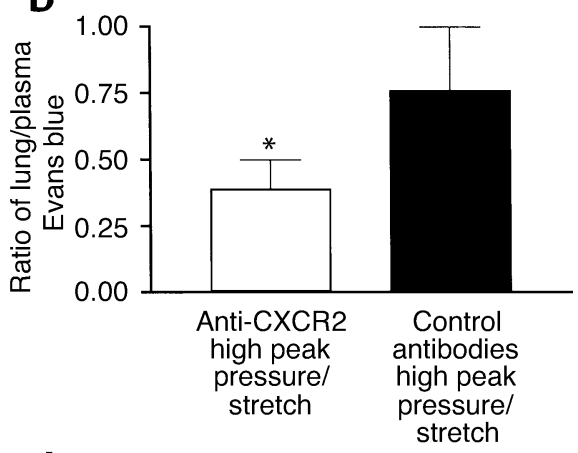

d

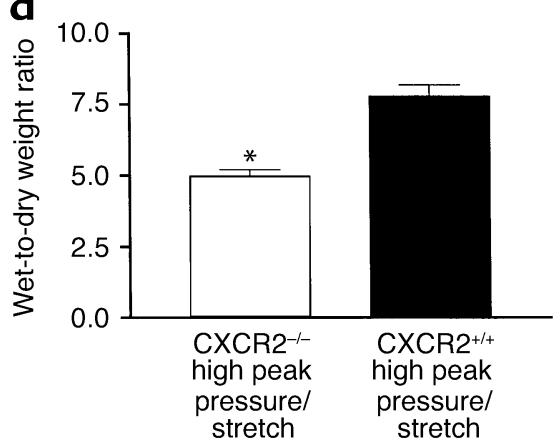

\section{Figure 10}

In vivo neutralization of murine CXCR2 attenuates VILI. (a) VILI scores of H\&Estained histopathologic sections from mice placed on high-peak pressure/ stretch ventilation protocol treated with neutralizing antibodies to CXCR2 or with control antibodies. A cumulative score was based on leukocyte infiltration, exudative edema, hemorrhage, and alveolar wall thickness $(n=15$; three random sections per lung and five lungs per group). ${ }^{*} P<0.05$. (b) Evans blue permeability index $(n=10$ mice per group). ${ }^{*} P<0.05$. (c) VILI scores of $\mathrm{H} \& \mathrm{E}$-stained histopathologic sections from CXCR2 ${ }^{-/}$mice placed on highpeak pressure/stretch ventilation protocol, as compared with $\mathrm{CXCR} 2^{+/+}$mice ( $n=15$; three random sections per lung and five lungs per group). ${ }^{*} P<0.05$. (d) Wet-to-dry weight ratio of lungs from $\mathrm{CXCR2}^{-/-}$and $\mathrm{CXCR} 2^{+/+}$mice placed on high-peak pressure/stretch ventilation protocol $(n=8$ mice per group). ${ }^{*} P<0.05$. has demonstrated that the increased respiratory rate used to prevent significant hypercarbia can lead to high levels of intrinsic positive end-expiratory pressure (PEEP), suggesting that the intrinsic PEEP may decrease lung injury secondarily to a decreased recruitment-derecruitment phenomenon (55). Previous data have demonstrated no intrinsic PEEP in mice placed on a Harvard Apparatus Co. ventilator, as long as the respiratory rate was less than 120 breaths per minute. Based on these studies, we chose a respiratory rate of 100 to ensure that the mice had complete exhalation during ventilation with an inspiratory-to-expiratory ratio of less than 1:1, thereby minimizing intrinsic PEEP (56). However, intrinsic PEEP in our experimental protocol was not measured; thus we cannot completely exclude the possibility that intrinsic PEEP was present and had some protective effect in our ventilated groups. We found, by Evans blue analysis of vascular permeability, that mice placed on a high-peak pressure/stretch protocol had greater lung injury than mice placed on the low-peak pressure/stretch protocol. Furthermore, the low-peak pressure/stretch group had more injury than the normal nonventilated mice. These results were confirmed by histopathological analysis, which demonstrated increased pulmonary edema, leukocyte infiltra-

\section{Figure 11}

Histopathology of lungs is associated with mechanical ventilation. Representative photomicrographs $(\times 400)$ with H\&E staining of the lung histopathology. (a) Mice placed on high-peak pressure/stretch mechanical ventilation treated with anti-murine CXCR2 or with control antibodies. (b) CXCR2-/- mice placed on high-peak pressure/ stretch mechanical ventilation, as compared with $\mathrm{CXCR} 2^{+/+}$mice.

tion, alveolar hemorrhage, and alveolar wall thickness (high-peak pressure/stretch >>> low-peak pressure/ stretch > normal nonventilated mice). These findings are consistent with other animal studies of VILI, which have established that high-peak/pressure mechanical ventilation induces diffuse alveolar edema, leukocyte infiltration, alveolar hemorrhage, basement membrane injury, and hyaline membrane formation $(7,8,30,31$, $54,57)$. Previous studies using rat models of VILI with or without preceding lung injury have elegantly demonstrated epithelial damage on transmission electron micrographs and increased plasma and lung edema markers specific for type I epithelial injury $(54,58)$.

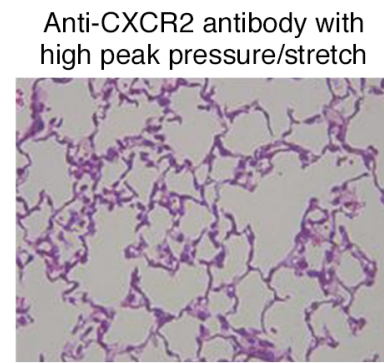

$\mathrm{CXCR}^{-/-}$mice with high peak pressure/stretch

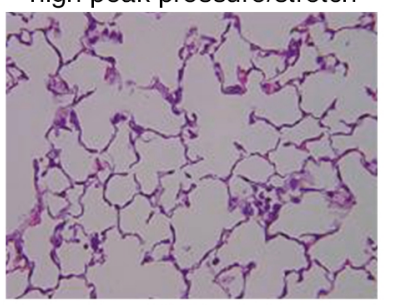

Six hours after mechanical ventilation
Control antibody with high peak pressure/stretch

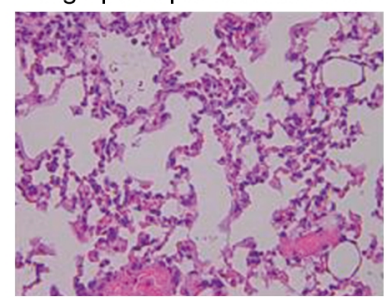

$\mathrm{CXCR}^{+/+}$mice with high peak pressure/stretch

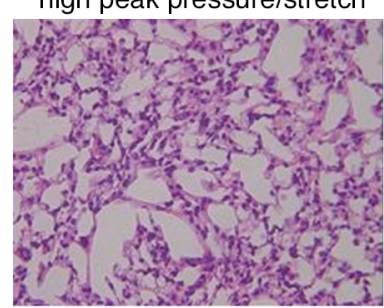




\section{Table 3}

Hemodynamic monitoring of normal nonventilated CXCR2 $2^{-/-}$mice, normal nonventilated CXCR2 $2^{+/+}$mice, and CXCR2 $2^{-/-}$mice and CXCR2 $2^{+/+}$ mice placed on a high-peak pressure/stretch mechanical ventilation protocol for 6 hours

\begin{tabular}{|c|c|c|c|c|c|c|c|c|c|c|c|}
\hline \multirow{2}{*}{\multicolumn{2}{|c|}{$\begin{array}{l}\text { Normal nonventilated } \\
\text { CXCR2 } 2^{-/-} \text {CXCR2 } 2^{+/+}\end{array}$}} & \multirow{2}{*}{\multicolumn{2}{|c|}{$\begin{array}{l}\text { Normal nonventilated } \\
\text { stretch CXCR2 } 2^{--}\end{array}$}} & \multicolumn{4}{|c|}{$\begin{array}{l}\text { High peak pressure/ } \\
\text { stretch CXCR2 } 2^{+/+}\end{array}$} & \multicolumn{4}{|c|}{ High peak pressure/ } \\
\hline & & & & \multicolumn{2}{|c|}{ First hour } & \multicolumn{2}{|c|}{ Sixth hour } & \multicolumn{2}{|c|}{ First hour } & \multicolumn{2}{|c|}{ Sixth hour } \\
\hline BP & Pulse & $\mathrm{BP}$ & Pulse & $\mathrm{BP}$ & Pulse & $\mathrm{BP}$ & Pulse & $\mathrm{BP}$ & Pulse & $\mathrm{BP}$ & Pulse \\
\hline $104 \pm 10$ & $492 \pm 16$ & $96 \pm 7$ & $485 \pm 15$ & $97 \pm 7$ & $484 \pm 9$ & $88 \pm 6$ & $496 \pm 12$ & $99 \pm 5$ & $501 \pm 7$ & $89 \pm 7$ & $511 \pm 14$ \\
\hline
\end{tabular}

Although we did not specifically measure epithelial cell injury, we speculate that there is epithelial injury in this murine model.

There is now abundant evidence suggesting that neutrophil sequestration into the lung is a pivotal event during the pathogenesis of ALI/ARDS (1,39-44). In addition, there are studies suggesting that VILI may promote and perpetuate ALI/ARDS (6,51-53). We demonstrated a significant increase in neutrophil sequestration in lungs from mice placed on a high-peak pressure/stretch as compared with a low-peak pressure/stretch mechanical ventilation protocol. There is evidence that the intrinsic pressure and volume generated during mechanical ventilation can cause enough shear stress injury to promote neutrophil infiltration into the lung $(8,30,31)$. Lungs taken from rabbits after 4 hours of mechanical ventilation, when studied under an electron microscope, demonstrated a diffuse infiltration of activated neutrophils (31). Taken together, our data and these studies exemplify that mechanical ventilation by itself can lead to neutrophil infiltration. However, the molecular and cellular mechanism(s) involved in recruiting these neutrophils remain to be elucidated.

Having demonstrated that the pathogenesis of VILI is associated with neutrophil sequestration, we next determined whether KC/CXCL1 and MIP-2/CXCL2/3, two potent CXC chemokine neutrophil chemoattractants, were elevated in the lungs during VILI. Both KC/CXCL1 expression and MIP-2/CXCL2/3 expression were significantly greater in the high-peak pressure/stretch group than in the low-peak pressure/stretch or normal nonventilated groups. In addition, the low-peak pressure/ stretch group had increased expression of KC/CXCL1 and MIP-2/CXCL2/3, whereas virtually no levels were found in the lungs from normal nonventilated mice. These results are similar to those of Tremblay and colleagues, who found elevated levels of MIP-2/CXCL2/3 in high-volume mechanically ventilated lungs of rats after 2 hours (59). Furthermore, our results extend the elegant in vitro studies that demonstrated increased IL-8 expression from alveolar macrophages, epithelial cells, or the combination of multiple pulmonary cell types cultured on an artificial plastic lung with continuous cyclic pressure-stretching (60-62). This suggests that high-peak pressure/stretch mechanical ventilation can induce the expression of ELR-positive CXC chemokines by deforming and/or injuring specific cell types.
$\mathrm{NF}-\kappa \mathrm{B}$ is a transcription factor that can modulate the expression of cytokines and chemokines during cellular stress and has been implicated in multiple inflammatory injuries to the lung (63). When a cell is in a resting state, NF- $\kappa \mathrm{B}$ is largely retained in the cytoplasm by a family of inhibitory proteins known as the I $\mathrm{KB}$ family. This family interacts with NF- $\kappa B$ to mask its nuclear translocation signal and sequester NF- $\kappa B$ proteins in the cytoplasm (64-66). I $\mathrm{KB} \alpha$ contains two serine residues at the $\mathrm{NH}_{2}$ terminus that regulate protein stability and a COOH-terminal PEST domain that contributes to basal protein turnover $(67,68)$. When the cell becomes stimulated by cytokines, mechanical stress, and LPS, serine residues in the $\mathrm{NH}_{2}$ terminus of I $\mathrm{I} B$ are phosphorylated. The I $\kappa \mathrm{B}$ is then ubiquitinated and is degraded by the $26 \mathrm{~S}$ protease, freeing $\mathrm{NK}-\kappa \mathrm{B}$ for translocation to the nucleus for transactivation of NF- $\kappa \mathrm{B}$ response genes (i.e., KC/CXCL1 and MIP-2/ CXCL2/3) (66). Furthermore, increased phosphorylation of $I \kappa B \alpha$ and degradation of $\mathrm{I} \kappa \mathrm{B} \alpha$ protein has been shown to correlate with NF- $\kappa B$ activation and transcription of CXC chemokines $(45,46)$. Based on these data, we determined whether NF- $\mathrm{KB}$ activity was a mechanism for increased expression of KC/CXCL1 and MIP-2/CXCL2/3. An increase in phosphorylation of $\mathrm{I} \kappa \mathrm{B} \alpha$ at an early time point, 2 hours after mechanical ventilation, was found. There was more phosphorylation of $I \kappa \mathrm{B} \alpha$ in the high-peak pressure/stretch than in the low-peak pressure/stretch mice. Confirming these results, we found a similar pattern of degraded I $\kappa \mathrm{B} \alpha$ protein, demonstrating its ubiquitination. Others have found that NF- $\mathrm{KB}$ is upregulated in response to stretch in both in vitro and ex vivo lung preparations $(69,70)$. Interestingly, at the 2-hour time point of mechanical ventilation, there was no increase in protein levels of IL-1 $\beta$ or TNF- $\alpha$ (data not shown). Together these studies suggest that mechanical stress promotes cellular activation of NF- $\kappa B$. While mechanical stress alone may be the primary promoter for activation of $\mathrm{NF}-\kappa \mathrm{B}$ and subsequent expression of KC/CXCL1 and MIP-2/ CXCL2/3, one cannot exclude that injury to the lung may be associated with a local hypoxic environment. Hypoxia has been shown to induce the expression of ELR-positive CXC chemokines $(71,72)$. Future studies will be useful to further delineate the contribution of mechanical stress and hypoxia in mediating the expression of KC/CXCL1 and MIP-2/CXCL2/3 during VILI. 
Patients with ALI/ARDS have an increased mortality related to multiorgan failure (1). Systemic inflammatory response or even traditional mechanical ventilation can contribute to overall mortality of these patients $(6,51-53)$. We determined whether $\mathrm{KC} /$ CXCL1 and MIP-2/CXCL2/3 expression was elevated in the serum from the mechanically ventilated groups, as compared with normal nonventilated mice. We did not find any significant differences in serum levels of $\mathrm{CXC}$ chemokine between groups. This suggests that $\mathrm{KC} / \mathrm{CXCL} 1$ and MIP-2/CXCL2/3 are playing a local compartmentalized role in the lung, thereby establishing a chemotactic gradient, which recruits neutrophils to the lung during VILI. Furthermore, the expression of CXCR2 in the lung and on leukocytes paralleled the production of both KC/CXCL1 and MIP-2/CXCL2/3 ligands and neutrophil sequestration during VILI. Similarly, other studies of inflammatory diseases, such as ALI resulting from hyperoxia in newborn rats, immune arthritis, psoriasis, and pneumonia, have demonstrated the importance of CXCR2 expression and its role in neutrophil recruitment during the pathogenesis of these diseases $(21,73-75)$. Collectively, these studies demonstrate that augmented levels of ELR-positive CXC chemokines are important in the recruitment of cells expressing CXCR2 during the pathogenesis of inflammatory diseases. Moreover, increased cell surface expression of CXCR2 was found on nonleukocyte cell populations in the high-peak pressure/stretch group. This implies that mechanical stimulation of nonleukocytes is involved in upregulating chemokine receptor expression. This is supported by other studies finding increased expression of CXCR2 on nonleukocytes such as fibroblasts and epithelial and endothelial cells (76-78), signifying a role for ligand/CXCR2 interactions that goes beyond neutrophil recruitment in mediating VILI.

Based on the above findings, we performed in vivo proof-of-concept studies to evaluate the direct role for KC/CXCL1 and MIP-2/CXCL2/3/CXCR2 ligands and their interaction with CXCR2 during the pathogenesis of VILI. We found that mice placed on the high-peak pressure/stretch protocol and treated with specific antibodies to KC/CXCL1, MIP-2/CXCL2/3, or KC/CXCL1 plus MIP-2/CXCL2/3 demonstrated significant reductions in VILI scores, as compared with mice placed on the same ventilator protocol and treated with appropriate control antibodies. Interestingly, neutralization of ligands $\mathrm{KC} /$ CXCL1 or MIP-2/CXCL2/3, or the combination of $\mathrm{KC} / \mathrm{CXCL} 1$ and MIP-2/CXCL2/3, gave similar reductions of VILI. This suggests the possibility of homologous desensitization of the receptor, whereby neutralization of $\mathrm{KC} / \mathrm{CXCL} 1$ may overexpose the receptor to MIP-2/ CXCL2/3 and vice versa, thereby resulting in desensitization of the receptor as is seen in chemotaxis assays at a high concentration of chemokine ligands $(79,80)$. Alternatively, this may also reflect the presence of other ELRpositive CXC chemokines (i.e., LIX/CXCR5 and Cka-3/ CXCL6) in the lung during VILI.
CXCR2 is the shared receptor not only for KC/CXCL1 and MIP-2/CXCL2/3 but also for LIX/CXCR5 and Cka3/CXCL6. To evaluate all ELR-positive CXC chemokine ligand interactions with CXCR2, mice were passively immunized with specific anti-murine CXCR2 antibodies and exposed to high-peak pressure/stretch ventilation protocol. The mice placed on the high-peak pressure/stretch protocol and treated with specific anti-murine CXCR2 antibodies demonstrated significant reductions in neutrophil sequestration as compared with mice placed on the same ventilator protocol and treated with control antibodies. Based on these findings, and knowing the central role of the neutrophil in most forms of ALI, we determined the effects of anti-murine CXCR2 on lung injury. Microvascular permeability analysis demonstrated that mice placed on the high-peak pressure/stretch protocol and treated with anti-murine CXCR2 had a marked reduction in vascular permeability. This reduction in microvascular permeability was confirmed by histopathologic assessment, which verified a marked decrease in leukocyte infiltration, pulmonary edema, alveolar hemorrhage, and alveolar wall thickness. We substantiated these results with a genetic approach by applying the same protocol to $\mathrm{CXCR}^{-/-}$mice. As in the anti-murine CXCR2-treated animals, we found significant reductions in leukocyte infiltration, pulmonary edema, alveolar hemorrhage, and alveolar wall thickness in CXCR2 $2^{-/}$mice, as determined by quantitative histopathologic analysis and lung wet-to-dry weight ratio. Furthermore, the VILI scores from CXCR2 $2^{-/-}$mice were significantly reduced as compared with those from the anti-KC/CXCL1, anti-MIP-2/CXCL2/3, or anti-KC/ CXCL1 + anti-MIP-2/CXCL2/3 groups. Therefore, inhibiting the receptor interactions with all ELR-positive CXC chemokine ligands has a greater effect on attenuating the pathogenesis of VILI. Our findings corroborate the finding of Kawano and associates (31). These investigators found that when neutrophils were depleted by nitrogen mustard there was a marked reduction in hyaline membrane formation and neutrophil infiltration, and only minimal presence of patchy necrosis (31). Together these studies demonstrate the importance of neutrophils during the genesis of VILI. Furthermore, our findings also demonstrate a population of cells that expresses CXCR2 during high-peak pressure/stretch VILI that are not leukocytes. Future studies will examine the role of these cells in mediating VILI.

In conclusion, we have demonstrated that $\mathrm{KC} /$ CXCL1 and MIP-2/CXCL2/3, through their interaction with their shared receptor CXCR2, play a pivotal role in mediating neutrophil recruitment and promoting VILI. This mechanism may perpetuate ALI/ARDS. In fact, ELR-positive CXC chemokines have been implicated for the recruitment of neutrophils to the lung during ALI/ARDS (13-20, 81). In addition, in some studies IL-8 was found to predict the onset and the outcome of ARDS (17-20). Moreover, IL-8 levels and neutrophils decline with time in patients who survive 
ARDS (16). Taken together, these findings suggest that CXCR2 and CXCR2 ligands are expressed during both VILI and the initiation of ALI/ARDS; therefore their additive or synergistic actions may, theoretically, lead to further pulmonary decompensation. The findings of this current study support the contention that CXCR2 and CXCR2 ligands mediate VILI, which may perpetuate the pathogenesis of ALI/ARDS.

\section{Acknowledgments}

This work was supported, in part, by NIH grants HL04493 (to J.A. Belperio); HL68694 and HL03906 (to M.P. Keane); P01HL67665 (to M.P. Keane and R.M. Strieter); and CA87879, P50CA90388, and HL66027 (to R.M. Strieter). J.A. Belperio is a recipient of the National and California State American Lung Association Research Grant Award (RG-019-B). M.P. Keane is a recipient of the American Lung Association Dalsemer Research Grant Award.

1. Ware, L.B., and Matthay, M.A. 2000. The acute respiratory distress syndrome. N. Engl. J. Med. 342:1334-1349.

2. Ranieri, V.M., et al. 1999. Effect of mechanical ventilation on inflammatory mediators in patients with acute respiratory distress syndrome: a randomized controlled trial. JAMA. 282:54-61.

3. Kollef, M.H., and Schuster, D.P. 1995. The acute respiratory distress syndrome. N. Engl. J. Med. 332:27-37.

4. Dreyfuss, D., Soler, P., Basset, G., and Saumon, G. 1988. High inflation pressure pulmonary edema. Respective effects of high airway pressure, high tidal volume, and positive end-expiratory pressure. Am. Rev. Respir. Dis. 137:1159-1164.

5. Corbridge, T.C., et al. 1990. Adverse effects of large tidal volume and low PEEP in canine acid aspiration. Am. Rev. Respir. Dis. 142:311-315.

6. 2000. Ventilation with lower tidal volumes as compared with traditional tidal volumes for acute lung injury and the acute respiratory distress syndrome. The Acute Respiratory Distress Syndrome Network. N. Engl. J. Med. 342:1301-1308.

7. Webb, H.H., and Tierney, D.F. 1974. Experimental pulmonary edema due to intermittent positive pressure ventilation with high inflation pressures. Protection by positive end-expiratory pressure. Am. Rev. Respir. Dis. 110:556-565.

8. Dreyfuss, D., and Saumon, G. 1998. Ventilator-induced lung injury: lessons from experimental studies. Am. J. Respir. Crit. Care Med. 157:294-323.

9. Bozic, C.R., et al. 1994. The murine interleukin 8 type B receptor homologue and its ligands. Expression and biological characterization. J. Biol. Chem. 269:29355-29358.

10. Lee, J., et al. 1995. Chemokine binding and activities mediated by the mouse IL-8 receptor. J. Immunol. 155:2158-2164.

11. Cerretti, D.P., et al. 1993. The murine homologue of the human interleukin-8 receptor type B maps near the Ity-Lsh-Bcg disease resistance locus. Genomics. 18:410-413.

12. Harada, A., et al. 1994. Cloning of a cDNA encoding a mouse homolog of the interleukin-8 receptor. Gene. 142:297-300.

13. Villard, J., et al. 1995. GRO alpha and interleukin-8 in Pneumocystis carinii or bacterial pneumonia and adult respiratory distress syndrome. Am.J. Respir. Crit. Care Med. 152:1549-1554.

14. Goodman, R.B., et al. 1996. Inflammatory cytokines in patients with persistence of the acute respiratory distress syndrome. Am. J. Respir. Crit. Care Med. 154:602-611.

15. Aggarwal, A., Baker, C.S., Evans, T.W., and Haslam, P.L. 2000. G-CSF and IL-8 but not GM-CSF correlate with severity of pulmonary neutrophilia in acute respiratory distress syndrome. Eur. Respir. J. 15:895-901.

16. Kurdowska, A., et al. 2001. Anti-interleukin 8 autoantibody: interleukin 8 complexes in the acute respiratory distress syndrome. Relationship between the complexes and clinical disease activity. Am. J. Respir. Crit. Care Med. 163:463-468.

17. Miller, E.J., et al. 1992. Elevated levels of NAP-1/interleukin-8 are present in the airspaces of patients with the adult respiratory distress syndrome and are associated with increased mortality. Am. Rev. Respir. Dis. 146:427-432.

18. Donnelly, S.C., et al. 1993. Interleukin-8 and development of adult respiratory distress syndrome in at-risk patient groups. Lancet. 341:643-647.
19. Chollet-Martin, S., et al. 1993. High levels of interleukin-8 in the blood and alveolar spaces of patients with pneumonia and adult respiratory distress syndrome. Infect. Immun. 61:4553-4559.

20. Meduri, G.U., et al. 1995. Inflammatory cytokines in the BAL of patients with ARDS. Persistent elevation over time predicts poor outcome. Chest. 108:1303-1314.

21. Mehrad, B., et al. 1999. CXC chemokine receptor-2 ligands are necessary components of neutrophil-mediated host defense in invasive pulmonary aspergillosis. J. Immunol. 163:6086-6094.

22. Moore, T.A., et al. 2000. Bacterial clearance and survival are dependent on CXC chemokine receptor- 2 ligands in a murine model of pulmonary Nocardia asteroides infection. J. Immunol. 164:908-915.

23. Tsai, W.C., et al. 2000. CXC chemokine receptor CXCR2 is essential for protective innate host response in murine Pseudomonas aeruginosa pneumonia. Infect. Immun. 68:4289-4296.

24. Greenberger, M.J., et al. 1996. Neutralization of macrophage inflammatory protein-2 attenuates neutrophil recruitment and bacterial clearance in murine Klebsiella pneumonia. J. Infect. Dis. 173:159-165.

25. Huffnagle, G.B., et al. 1995. The role of monocyte chemotactic protein1 (MCP-1) in the recruitment of monocytes and CD4+ T cells during a pulmonary Cryptococcus neoformans infection. J. Immunol. 155:4790-4797.

26. Huffnagle, G.B., et al. 1997. Macrophage inflammatory protein-1alpha (MIP-1alpha) is required for the efferent phase of pulmonary cell-mediated immunity to a Cryptococcus neoformans infection. J. Immunol. 159:318-327.

27. Keane, M.P., et al. 1999. Neutralization of the CXC chemokine, macrophage inflammatory protein-2, attenuates bleomycin-induced pulmonary fibrosis. J. Immunol. 162:5511-5518.

28. Belperio, J.A., et al. 2000. The role of the CC chemokine, RANTES, in acute lung allograft rejection. J. Immunol. 165:461-472.

29. Olszyna, D.P., et al. 2001. CXC chemokine receptor 2 contributes to host defense in murine urinary tract infection. J. Infect. Dis. 184:301-307.

30. Imanaka, H., et al. 2001. Ventilator-induced lung injury is associated with neutrophil infiltration, macrophage activation, and TGF-beta 1 mRNA upregulation in rat lungs. Anesth. Analg. 92:428-436.

31. Kawano, T., et al. 1987. Effect of granulocyte depletion in a ventilated surfactant-depleted lung. J. Appl. Physiol. 62:27-33.

32. Colletti, L.M., et al. 1995. Chemokine expression during hepatic ischemia/reperfusion-induced lung injury in the rat. The role of epithelial neutrophil activating protein. J. Clin. Invest. 95:134-141.

33. Nishina, K., et al. 1998. Intravenous lidocaine attenuates acute lung injury induced by hydrochloric acid aspiration in rabbits. Anesthesiology. 88:1300-1309.

34. Green, T.P., Johnson, D.E., Marchessault, R.P., and Gatto, C.W. 1988. Transvascular flux and tissue accrual of Evans blue: effects of endotoxin and histamine. J. Lab. Clin. Med. 111:173-183.

35. Saria, A., and Lundberg, J.M. 1983. Evans blue fluorescence: quantitative and morphological evaluation of vascular permeability in animal tissues. J. Neurosci. Methods. 8:41-49.

36. Chumakov, K.M. 1994. Reverse transcriptase can inhibit PCR and stimulate primer-dimer formation. PCR Methods Appl. 4:62-64.

37. Belperio, J.A., et al. 2001. Critical role for the chemokine MCP-1/CCR2 in the pathogenesis of bronchiolitis obliterans syndrome. J. Clin. Invest. 108:547-556. doi:10.1172/JCI200112214.

38. Belperio, J.A., et al. 2002. Critical role for CXCR3 chemokine biology in the pathogenesis of Bronchiolitis obliterans syndrome. J. Immunol. 169:1037-1049.

39. Lee, W.L., and Downey, G.P. 2001. Neutrophil activation and acute lung injury. Curr. Opin. Crit. Care. 7:1-7.

40. Rinaldo, J.E. 1986. Mediation of ARDS by leukocytes. Clinical evidence and implications for therapy. Chest. 89:590-593.

41. Steinberg, K.P., et al. 1994. Evolution of bronchoalveolar cell populations in the adult respiratory distress syndrome. Am. J. Respir. Crit. Care Med. 150:113-122.

42. Folz, R.J., Abushamaa, A.M., and Suliman, H.B. 1999. Extracellular superoxide dismutase in the airways of transgenic mice reduces inflammation and attenuates lung toxicity following hyperoxia. J. Clin. Invest. 103:1055-1066.

43. Kawabata, K., et al. 2000. Delayed neutrophil elastase inhibition prevents subsequent progression of acute lung injury induced by endotoxin inhalation in hamsters. Am. J. Respir. Crit. Care Med. 161:2013-2018.

44. Thommasen, H.V., Russell, J.A., Boyko, W.J., and Hogg, J.C. 1984. Transient leucopenia associated with adult respiratory distress syndrome. Lancet. 1:809-812.

45. Shattuck-Brandt, R.L., and Richmond, A. 1997. Enhanced degradation of I-kappaB alpha contributes to endogenous activation of NF-kappaB in Hs294T melanoma cells. Cancer Res. 57:3032-3039.

46. Devalaraja, M.N., Wang, D.Z., Ballard, D.W., and Richmond, A. 1999. Elevated constitutive IkappaB kinase activity and IkappaB-alpha phosphorylation in Hs294T melanoma cells lead to increased basal MGSA/GRO-alpha transcription. Cancer Res. 59:1372-1377. 
47. Yang, J., and Richmond, A. 2001. Constitutive IkappaB kinase activity correlates with nuclear factor-kappaB activation in human melanoma cells. Cancer Res. 61:4901-4909.

48. Weiland, J.E., et al. 1986. Lung neutrophils in the adult respiratory distress syndrome. Clinical and pathophysiologic significance. Am. Rev. Respir. Dis. 133:218-225.

49. Martin, T.R., Pistorese, B.P., Hudson, L.D., and Maunder, R.J. 1991. The function of lung and blood neutrophils in patients with the adult respiratory distress syndrome. Implications for the pathogenesis of lung infections. Am. Rev. Respir. Dis. 144:254-262.

50. Mead, J., Takishima, T., and Leith, D. 1970. Stress distribution in lungs: a model of pulmonary elasticity. J. Appl. Physiol. 28:596-608.

51. Hickling, K.G., Henderson, S.J., and Jackson, R. 1990. Low mortality associated with low volume pressure limited ventilation with permissive hypercapnia in severe adult respiratory distress syndrome. Intensive Care Med. 16:372-377.

52. Amato, M.B., et al. 1995. Beneficial effects of the "open lung approach" with low distending pressures in acute respiratory distress syndrome. A prospective randomized study on mechanical ventilation. Am. J. Respir. Crit. Care Med. 152:1835-1846.

53. Amato, M.B., et al. 1998. Effect of a protective-ventilation strategy on mortality in the acute respiratory distress syndrome. N. Engl. J. Med. 338:347-354

54. Dreyfuss, D., Basset, G., Soler, P., and Saumon, G. 1985. Intermittent positive-pressure hyperventilation with high inflation pressures produces pulmonary microvascular injury in rats. Am. Rev. Respir. Dis. 132:880-884.

55. de Durante, G., et al. 2002. ARDSNet lower tidal volume ventilatory strategy may generate intrinsic positive end-expiratory pressure in patients with acute respiratory distress syndrome. Am. J. Respir. Crit. Care Med. 165:1271-1274.

56. Volgyesi, G.A., Tremblay, L.N., Webster, P., Zamel, N., and Slutsky, A.S 2000. A new ventilator for monitoring lung mechanics in small animals. J. Appl. Physiol. 89:413-421.

57. Kolobow, T., et al. 1987. Severe impairment in lung function induced by high peak airway pressure during mechanical ventilation. An experimental study. Am. Rev. Respir. Dis. 135:312-315.

58. Frank, J.A., et al. 2002. Low tidal volume reduces epithelial and endothelial injury in acid-injured rat lungs. Am. J. Respir. Crit. Care Med. 165:242-249.

59. Tremblay, L., Valenza, F., Ribeiro, S.P., Li, J., and Slutsky, A.S. 1997. Injurious ventilatory strategies increase cytokines and c-fos m-RNA expression in an isolated rat lung model. J. Clin. Invest. 99:944-952.

60. Pugin, J., et al. 1998. Activation of human macrophages by mechanical ventilation in vitro. Am. J. Physiol. 275:L1040-L1050.

61. Vlahakis, N.E., Schroeder, M.A., Limper, A.H., and Hubmayr, R.D. 1999 Stretch induces cytokine release by alveolar epithelial cells in vitro. Am. J. Physiol. 277:L167-L173.

62. Mourgeon, E., et al. 2000. Mechanical stretch stimulates macrophage inflammatory protein-2 secretion from fetal rat lung cells. Am. J. Physiol. Lung Cell. Mol. Physiol. 279:L699-L706.

63. Blackwell, T.S., and Christman, J.W. 1997. The role of nuclear factor-kappa
B in cytokine gene regulation. Am. J. Respir. Cell Mol. Biol. 17:3-9.

64. Beg, A.A., et al. 1992. I kappa B interacts with the nuclear localization sequences of the subunits of NF-kappa B: a mechanism for cytoplasmic retention. Genes Dev. 6:1899-1913.

65. Beg, A.A., Finco, T.S., Nantermet, P.V., and Baldwin, A.S., Jr. 1993. Tumor necrosis factor and interleukin-1 lead to phosphorylation and loss of I kappa B alpha: a mechanism for NF-kappa B activation. Mol. Cell. Biol. 13:3301-3310.

66. Baldwin, A.S., Jr. 1996. The NF-kappa B and I kappa B proteins: new discoveries and insights. Annu. Rev. Immunol. 14:649-683.

67. Maniatis, T. 1997. Catalysis by a multiprotein IkappaB kinase complex. Science. 278:818-819.

68. Rodriguez, M.S., Michalopoulos, I., Arenzana-Seisdedos, F., and Hay, R.T. 1995. Inducible degradation of I kappa B alpha in vitro and in vivo requires the acidic C-terminal domain of the protein. Mol. Cell. Biol. 15:2413-2419.

69. Lentsch, A.B., Czermak, B.J., Bless, N.M., Van Rooijen, N., and Ward, P.A. 1999. Essential role of alveolar macrophages in intrapulmonary activation of NF-kappaB. Am. J. Respir. Cell Mol. Biol. 20:692-698.

70. Held, H.D., Boettcher, S., Hamann, L., and Uhlig, S. 2001. Ventilationinduced chemokine and cytokine release is associated with activation of nuclear factor-kappaB and is blocked by steroids. Am. J. Respir. Crit. Care Med. 163:711-716.

71. Metinko, A.P., Kunkel, S.L., Standiford, T.J., and Strieter, R.M. 1992. Anoxia-hyperoxia induces monocyte-derived interleukin-8. J. Clin. Invest. 90:791-798

72. Karakurum, M., et al. 1994. Hypoxic induction of interleukin-8 gene expression in human endothelial cells. J. Clin. Invest. 93:1564-1570.

73. Auten, R.L., et al. 2001. Nonpeptide CXCR2 antagonist prevents neutrophil accumulation in hyperoxia-exposed newborn rats. J. Pharmacol. Exp. Ther. 299:90-95.

74. Schimmer, R.C., et al. 1997. Streptococcal cell wall-induced arthritis. Requirements for neutrophils, P-selectin, intercellular adhesion molecule1, and macrophage-inflammatory protein-2.J. Immunol. 159:4103-4108.

75. Kulke, R., et al. 1998. The CXC receptor 2 is overexpressed in psoriatic epidermis. J. Invest. Dermatol. 110:90-94.

76. Nirodi, C.S., et al. 2000. Chemokine and chemokine receptor expression in keloid and normal fibroblasts. Wound Repair Regen. 8:371-382.

77. Williams, E.J., et al. 2000. Distribution of the interleukin-8 receptors, CXCR1 and CXCR2, in inflamed gut tissue. J. Pathol. 192:533-539.

78. Addison, C.L., et al. 2000. The CXC chemokine receptor 2, CXCR2, is the putative receptor for ELR + CXC chemokine-induced angiogenic activity. J. Immunol. 165:5269-5277.

79. Keane, M.P., et al. 2001. ENA-78 is an important angiogenic factor in idiopathic pulmonary fibrosis. Am. J. Respir. Crit. Care Med. 164:2239-2242.

80. Ben-Baruch, A., Michiel, D.F., and Oppenheim, J.J. 1995. Signals and receptors involved in recruitment of inflammatory cells. J. Biol. Chem. 270:11703-11706

81. Goodman, D.J., Von Albertini, M., Willson, A., Millan, M.T., and Bach, F.H 1996. Direct activation of porcine endothelial cells by human natural killer cells. Transplantation. 61:763-771. 